\title{
Controlling light harvesting with light
}

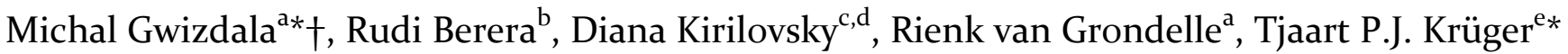 \\ a Department of Physics and Astronomy, VU Amsterdam, 1081 HV Amsterdam, The Netherlands. \\ E-mails: m.s.gwizdala@vu.nl and r.van.grondelle@vu.nl \\ ${ }^{\mathrm{b}}$ Department of Food Sciences, Faculty of Agriculture, Kagawa University, Miki-cho, Kagawa 761-0795, Japan \\ E-mail: r.berera@vu.nl \\ ${ }^{c}$ Centre National de la Recherche Scientifique (CNRS), I2BC, UMR 9198, 91191 Gif-sur-Yvette, France ${ }^{\mathrm{d}}$ Commissariat \\ à l'Energie Atomique (CEA), Institut de Biologie et Technologies de Saclay (iBiTec-S), 91191 Gif-sur-Yvette, France \\ E-mail: diana.kirilovsky@cea.fr \\ ${ }^{\text {e }}$ Department of Physics, University of Pretoria, oo28 Hatfield, South Africa \\ E-mail: tjaart.kruger@up.ac.za
}

Photosynthesis, Excitation Energy Flow Regulation, Light Harvesting Complexes, Cyanobacteria, Phycobilisomes, Single Molecule Spectroscopy, Fluorescence blinking

\begin{abstract}
When exposed to intense sunlight all organisms performing oxygenic photosynthesis implement various photoprotective strategies to prevent potentially lethal photodamage. The rapidly responding photoprotective mechanisms, occurring in the light-harvesting pigment-protein antennae, take effect within tens of seconds, while the dramatic and potentially harmful light intensity fluctuations manifest also on shorter timescales. Here we show that upon illumination, individual phycobilisomes from Synechocystis PCC 6803, which in vivo under low-light conditions harvest solar energy, have the inbuilt capacity to switch rapidly and reversibly into light-activated energy-dissipating states. Simultaneously measured fluorescence intensity, lifetime and spectra, compared with a multi-compartmental kinetic model, revealed that essentially any subunit of a phycobilisome can be quenched, and that the core complexes were targeted most frequently. Our results provide the first evidence for fluorescence blinking from a biologically active system at physiological light intensities and suggest that the light-controlled switches to intrinsically available energy dissipating states are responsible for a novel type of photoprotection in cyanobacteria. We anticipate other photosynthetic organisms to employ similar strategies to respond instantly to rapid solar light intensity fluctuations. A detailed understanding of the photophysics of photosynthetic antenna complexes is of great interest for bioinspired solar energy technologies.
\end{abstract}

\section{INTRODUCTION}

One important design principle of the photosynthetic apparatus is the large ratio of antenna pigments to reaction center pigments. ${ }^{1-3}$ This ensures effective absorption of dim light and introduces the possibility to adapt swiftly to changing light conditions, in particular to a sudden increase in light intensity. The latter functionality is achieved through complex mechanisms that regulate the probability of non-radiative decay of electronic excitations in the antenna network of oxygenic photosynthetic organisms (i.e. plants, algae and cyanobacteria) and is known as $\mathrm{qE}$, the energy-dependent, rapidly reversible component of photoprotection that can be observed as nonphotochemical quenching (NPQ) of chlorophyll a fluorescence (see Ref. 2 for recent reviews). As such, excess absorbed energy, which would give rise to harmful by-products, ${ }^{4}$ is safely dissipated as heat and a sufficient energy throughput is maintained at the reaction center to balance the rate of photochemistry.

While $\mathrm{qE}$ involves specific and controlled energy dissipation pathways, it is also known that upon illumination, essentially every natural and synthetic nanoscale emitter switches randomly and reversibly to intrinsic, metastable dark states, during which absorbed energy is thermally dissipated, a phenomenon known as fluorescence intermittency or blinking. ${ }^{5}$ It was recently attempted to associate fluorescence blinking with photoprotection, ${ }^{6,7}$ yet all reported studies on individual photosynthetic lightharvesting complexes have been performed using an excitation fluence that was several orders of magnitude higher than sunlight, potentially inducing effects that are highly unexpected for biological complexes under physiological conditions. For example, the repetitive excitations in those studies give rise to significant singlet-triplet an- 
nihilation, ${ }^{8,9}$ the enhanced thermal dissipation of which may increase protein conformational diffusion and hence also fluorescence blinking. Alternatively, a higher excitation rate may lead to the formation of long-living radical dark states, a key photoproduct responsible for blinking of isolated fluorophores. ${ }^{10}$

The initial step in exploring a possible relationship between photoprotection and blinking requires a singlemolecule study at physiological light intensities, i.e. under conditions that well-represent the natural environment. In ensemble approaches, the stochastic behavior of blinking is averaged to a single intensity value and physiologically relevant energy dissipation cannot easily be discriminated from irreversible photobleaching. A study of phycobilisomes (PBs), the largest antenna complex known to be involved with NPQ, allowed us to use physiological excitation intensities and still resolve the photodynamics at the single antenna level (vide infra). PB is the peripheral, multi-subunit, extra-membranous antenna complex of cyanobacteria and red algae. In Synechocystis PCC 6803 (hereafter Synechocystis), the subunits of PBs are arranged as six rods radiating from a three-cylindrical core (Fig. 1a). ${ }^{11-13}$ The rods and core are composed of phycobiliproteins, covalently binding phycobilins (i.e. linear tetrapyrroles) and mostly pigment-free linker proteins. ${ }^{1,14}$ Each core cylinder is composed of four allophycocyanin (APC) trimers, each binding six phycobilins, giving 72 pigments per core. The two basal cylinders contain trimers with subunits substituted by the so-called terminal emitters (Fig. 1a, red), resulting in a fluorescence shift from $660 \mathrm{~nm}$ to $\sim 680 \mathrm{~nm}{ }^{15}$ These terminal emitters $(6$ pigments per core; collectively referred to as APC68o) transfer the excitation energy from the other core subunits (66 pigments per core; collectively named APC66o; Fig. 1a, light blue) to the photosystems containing the photosynthetic reaction centers. ${ }^{11,15}$ The rods are composed of, on average, two to three hexamers of phycocyanin (PC), where each hexamer binds 18 phycobilins that fluoresce at $\sim 650 \mathrm{~nm}$ (Fig. 1a, dark blue). A whole Synechocystis PB complex contains up to 396 phycobilin pigments.

The recently discovered NPQ mechanism involving PB depends on a light-induced conformational change in a water-soluble Orange Carotenoid Protein (OCP $)^{16-18}$ and its subsequent binding to the core of $\mathrm{PB},{ }^{19,20}$ upon which an energy trap is created at the APC660. ${ }^{21,22}$ Each of these steps costs time and from the moment an organism is exposed to high intensity light it takes at least several seconds before OCP-mediated quenching reaches its full capacity. ${ }^{16,19}$ During this time, deleterious photoproducts can be formed unless another energy dissipating mechanism is instantly activated. Single-molecule spectroscopy provides a unique lens to investigate the possible existence of such a fast mechanism: due to the required speed of activation, such a mechanism is expected to be intrinsic to the $\mathrm{PB}$ and reflected in the fluorescence blinking pattern when increasing the excitation intensity.

\section{RESULTS}

Remarkably, using excitation intensities corresponding to the intensity of sunlight reaching the Earth (i.e. typically $<2000 \mu \mathrm{mol}$ photons $\mathrm{m}^{-2} \mathrm{~s}^{-1}$ or $<80.7 \mathrm{~mW} \mathrm{~cm}^{-2}$ ), various reversible intensity transitions to metastable, weakly emissive states were observed (e.g. Fig. 1b-d at $\sim 111, \sim 222$, and $\sim 1174 \mu \mathrm{mol}$ photons $\mathrm{m}^{-2} \mathrm{~s}^{-1}$, respectively). Many intensity decreases were large and abrupt, characteristic of fluorescence blinking. The observation of blinking from such a large complex was quite surprising and indicates that the PB subunits were well-connected. Additionally, diffusion-limited excitation energy transfer kinetics explains why the fluorescence did not decrease to the background level. These complexes are, to our knowledge, the largest biologically active systems yet observed to display blinking.
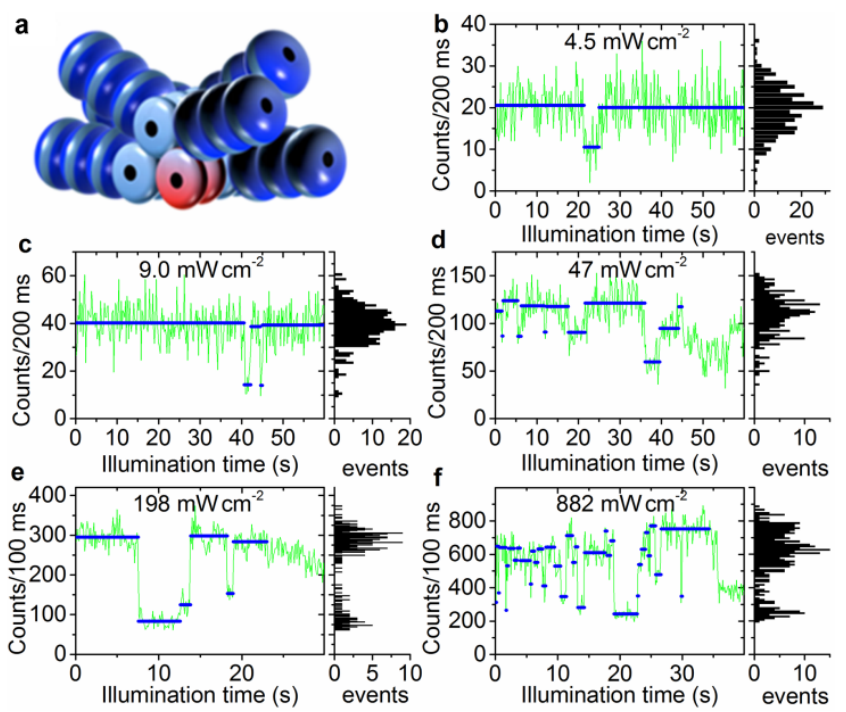

Figure 1. Single PB fluorescence intensity dynamics under different light intensities.

a, Structure of Synechocystis PB as described in detail in the text and redrawn after Ref. 12. PC hexamers are dark blue, APC66o trimers are light blue and APC68o trimers are red. b-f, Examples of fluorescence intensity traces (green), each measured under illumination at a distinct excitation intensity (indicated on top). For sections before photodegradation (see text), resolved intensity levels are indicated in blue and intensity distribution is shown on the right.

The examples displayed in Fig. $1 \mathrm{~b}-\mathrm{f}$ show that the intensity fluctuation rate and distribution depend on the excitation intensity. To further study the character and potential role of these intensity dynamics, we first used a sufficiently high excitation intensity to resolve the fluorescence intensities with $10-\mathrm{ms}$ resolution while simultaneously recording the fluorescence spectra (specifically, 1174 $\mathrm{mW} \mathrm{cm}^{-2}$, corresponding to $\sim 29$, $000 \mu \mathrm{mol}$ photons $\mathrm{m}^{-2} \mathrm{~s}^{-1}$ ). In addition, the fluorescence photons were time tagged to provide fluorescence lifetime information.

Prior to further analysis it was important to screen the data to ensure that the size and integrity of PB did not 
vary before and throughout a measurement. Due to the limited stability of isolated $\mathrm{PB}^{23}$ a significant heterogeneity in size and number of bound pigments was expected. For each complex, the slope of a linear fit to the lifetimeintensity distribution (Fig. 2b) was used as the primary measure of its size. In general, the direct proportionality between the fluorescence lifetime and intensity indicates a varying probability for non-radiative decay that is due to the action of a quencher on one of the subunits of a wellconnected complex. ${ }^{24}$ The core subunits constitute the primary sites of fluorescence emission and hence the interactions within the core largely determine the fluorescence lifetime of PB. In contrast, quenching of a rod subunit signifies a strongly non-equilibrated system and is therefore expected to affect the observed fluorescence intensity more significantly than the lifetime, thus increasing the lifetime-intensity slope.
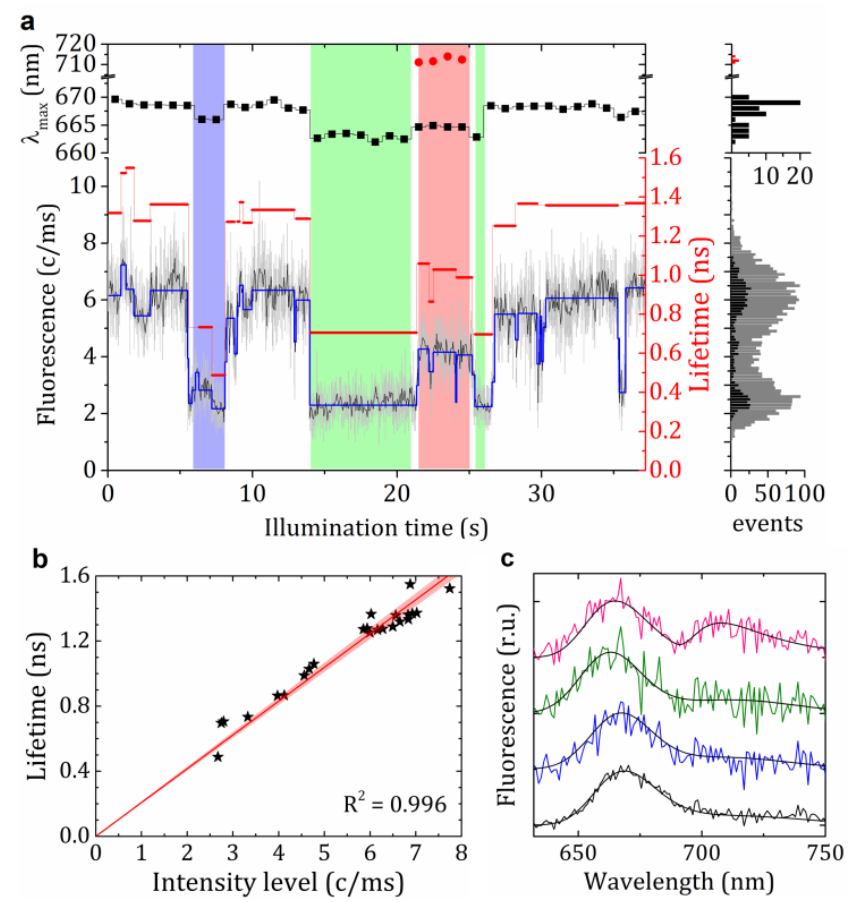

Figure 2. Representative fluorescence fluctuations from a single PB complex.

a, Trace of binned fluorescence counts (bottom black and light-gray), associated lifetimes (red) and associated peak positions of 1-s binned spectra ( $\lambda_{\max }$, black squares and red circles). The intensity is displayed in 6o-ms (black) and 10ms (light-gray) bins. The associated intensity and spectral peak distributions are shown on the right. Resolved intensity levels are shown by horizontal blue lines. Fluorescence lifetimes were determined for data corresponding to each intensity level (see Supporting Experimental Information for details). Four quenched states are indicated by shaded regions and correspond to a small blue-shift (blue), larger blue-shift (green) or strongly red-shifted emission near $712 \mathrm{~nm}$ (red). An excitation intensity of $1174 \mathrm{~mW} \mathrm{~cm}^{-2}$ was used. b, Fluorescence intensity-lifetime correlation for the resolved levels in a, together with a linear fit (red line, with the $2 \sigma$ confidence band shaded). Coefficient of determination of the fit is shown at bottom right. c, Emission spectra corresponding to the average of each color-coded, shaded region in a. Bottom spectrum is the average emission during the first $5 \mathrm{~s}$ and is typical for unquenched states. Spectra are normalized and offset for clarity. Black lines show fits. See Supporting Experimental Information for details.

Such reversible increases in the slope were rather uncommon (vide infra) and therefore did not significantly affect the overall slope. For example, a reversible increase in the lifetime-intensity ratio occurs in Fig. 2a between the second and third resolved levels and corresponds to anti-correlated shifts in the lifetime and intensity. Moreover, the small fraction of uncorrelated shifts (e.g., near the end of the trace in Fig. 2a) also kept the uncertainty in the linear fit sufficiently small. However, numerous complexes were found for which the slope increased irreversibly at a particular moment during the measurement (e.g., Fig. S2). The latter phenomenon can be best explained by detachment or bleaching of subunits, both processes of which reduce the effective absorption cross-section of a complex but affect the fluorescence lifetime little or negligibly. ${ }^{25,26}$ Such photodegradation occurred frequently when using relatively high excitation intensities and was often accompanied by irreversible blue-shifted emission. Moreover, the distribution of slopes among complexes that did not photodegrade during the measurement also varied, suggesting that numerous complexes had lost some functional subunits before the onset of illumination. We assumed that intact PBs correspond to units containing nearly 400 pigments, arranged as described above, and displayed the highest fluorescence intensity observed from an experimental batch of individually measured PBs. This assumption was confirmed by the agreement between experimental data and modeling shown in Fig. 3 (vide infra) as well as the agreement between the calculated and measured fluorescence intensities (Fig. S3). Overall for further investigation, we used only sections of traces before photodegradation and excluded complexes showing light-independent degradation. The average survival time corresponding to different excitation intensities is shown in Table $\mathrm{S}$, indicating that a considerable fraction of complexes degraded in the absence of light, i.e., before the onset of illumination.

Figs. 2 and $\mathrm{S} 1$ show a typical fluorescence trace from a complex that did not undergo photodegradation but demonstrated distinct reversible switches into quenched states accompanied by three different spectroscopic changes. The blue-shifted emission featured as a shift of the whole spectrum, while the red-shifted emission appeared as an additional band (Fig. 2c; Fig. Sib). Only a fraction of complexes ( $\sim 5 \%)$ exhibited red-shifted emission, while essentially all other quenched states were typified by a blue-shift. Curiously, the red-shifted emission in Fig. 2a was preceded and succeeded by a blue-shifted quenched state. Inspection of 38 red states and their accompanying 10-ms binned intensity traces indicates that $74 \%$ of the states exhibiting red emission were preceded 
by a blue-shifted quenched state, while $55 \%$ were followed by such a state.

The time scale of intensity dynamics (ms - s) and associated relatively small blue spectral shifts suggests that local protein conformational changes are involved in the access and stabilization of the quenched states. It was previously demonstrated that relatively slow conformational changes in the vicinity of an excitation energy trap can quantitatively explain fluorescence blinking in the main plant light-harvesting complex, ${ }^{7}$ a mechanism that was proposed to apply to pigment-protein complexes in general. Indeed, in our measurements, using a crosslinker to rigidify the protein scaffold led to a sizable decrease in the intensity switching rates, as illustrated in Table 1 for isolated rod fractions. ${ }^{27}$

Table 1. Intensity switching rates of individual rod fractions treated with different concentrations of glutaraldehyde (GA).

\begin{tabular}{ccc} 
\%GA & $\boldsymbol{k}_{U \rightarrow Q}\left(\mathrm{~s}^{-1}\right)$ & $\boldsymbol{k}_{Q \rightarrow U}\left(\mathrm{~s}^{-1}\right)$ \\
\hline 0.0 & $0.65 \pm 0.11$ & $0.73 \pm 0.08$ \\
0.5 & $0.29 \pm 0.03$ & $0.50 \pm 0.06$ \\
1.0 & $0.07 \pm 0.02$ & $0.18 \pm 0.03$
\end{tabular}

PC rate of switching into a lower intensity state $\left(k_{U \rightarrow Q}\right)$ and into a higher intensity state $\left(k_{Q \rightarrow U}\right)$, determined as for the whole PB complex in Fig. 4a. Data screening was performed similarly to intact $\mathrm{PB}$, so that only the largest rods were analyzed, leaving an average of 37 complexes for each of the three data sets. Complexes were measured for $60 \mathrm{~s}$ each at an excitation intensity of $2940 \mathrm{~mW} \mathrm{~cm}^{-2}$. Time bins of $50 \mathrm{~ms}$ were used.

To identify the possible sites of reversible fluorescence quenching, we investigated the corresponding spectroscopic changes by constructing compartmental kinetic models, considering all pigments emitting at a similar wavelength to belong to the same pigment pool (see Supporting Calculations). For intact PBs, the experimental intensity-spectral relationship followed the theoretical predictions well (Fig. 3). A similar agreement between the experimental and theoretical results was observed for the smaller PB complexes isolated from mutants (Fig. S4). ${ }^{28,29}$

It appears that quenching of a core unit (Fig. 3, black and blue curves) occurred more frequently than quenching of a PC subunit (Fig. 3, green curve) and that APC68o was quenched more frequently than APC66o (Fig S4a), qualitatively following the relative probability of finding an excitation in each of the pigment pools. We conclude that in the intact complex, essentially any type of subunit can switch to a quenched state, thus providing multiple channels in PB for excitation energy dissipation. This conclusion agrees with the observation of blinking from individual, isolated rods ${ }^{27}$ and core units ${ }^{29}$ (Table 1 and Fig. S4a, respectively) and individual APC trimers. ${ }^{26,30}$

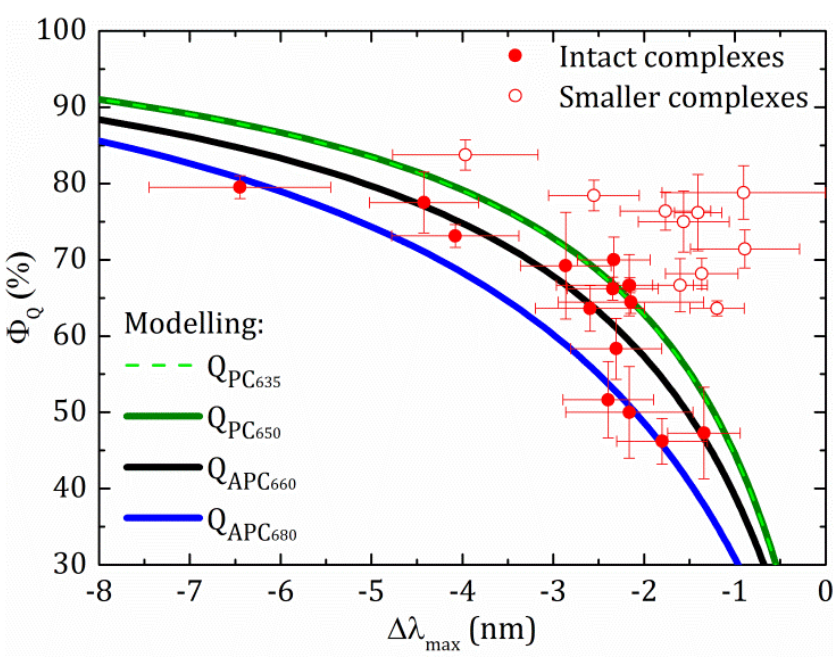

Figure 3. Quantum yield of fluorescence quenching vs. fluorescence peak position shift upon entering a quenched state.

Data points for complexes that underwent reversible fluorescence quenching (filled circles) follow theoretical prediction (lines), where each colored line corresponds to quenching of a different pigment pool (see Supporting Calculations). Complexes not recovering completely after quenching or associated with a sub-maximum intensity before quenching are denoted by open circles. Each data point corresponds to a distinct switching event. Only data with an uncertainty on $\Delta \lambda_{\max }$ smaller than $2 \mathrm{~nm}$ is shown, involving 24 complexes. An excitation intensity of $882 \mathrm{~mW} \mathrm{~cm}^{-2}$ was used. Error bars denote standard deviations. See Supporting Experimental Information for more information.

The degree to which the intensity switches are lightdriven was explored by varying the excitation intensity in the range from $1174 \mathrm{~mW} \mathrm{~cm}^{-2}$ down to $4.5 \mathrm{~mW} \mathrm{~cm}^{-2}$ (equivalently, $\sim 30$,ooo down to $\sim 111 \mu \mathrm{mol}$ photons $\mathrm{m}^{-2} \mathrm{~s}^{-1}$ ), the lower limit of which represents an unprecedentedly low intensity for single-molecule studies. The switching frequency between unquenched and quenched states showed a strong light dependence, and the average dwell time in all intensity states exhibited a light-dependent shortening (Fig. S5), which is particularly pronounced for the unquenched states (Fig. S5a). From Fig. S5a and b we calculated the rates of switching into and out of a quenched state, i.e., $k_{U \rightarrow Q}$ and $k_{O \rightarrow U}$, respectively, for each excitation intensity (Fig. 4a). Both rates increase with the excitation intensity, suggesting strongly light-activated transitions. Meaningfully, $k_{U \rightarrow Q}$ shows the largest increase (Fig. 4 b and Fig. S6), indicating that in particular the formation of quenched states is driven under high light intensities, and thermal energy dissipated during quenching probably also plays a role in triggering the back-switch. Moreover, the ratio of the switching rates (i.e., $k_{U \rightarrow Q} /$ $\left.k_{Q \rightarrow U}\right)$ can be approximated by a logarithmic dependence on the excitation intensity (Fig. $4 \mathrm{~b}$ and Fig. S6), indicating a particularly strong light dependence of $k_{U \rightarrow Q}$ for the lowest measured intensities (up to $\sim 100 \mathrm{~mW} \mathrm{~cm}^{-2} \approx 2500$ $\mu \mathrm{mol}$ photons $\left.\mathrm{m}^{-2} \mathrm{~s}^{-1}\right)$. The equilibrium shift to quenched 
states resulting from increasing excitation is further illustrated by the intensity histograms in Fig. S7.

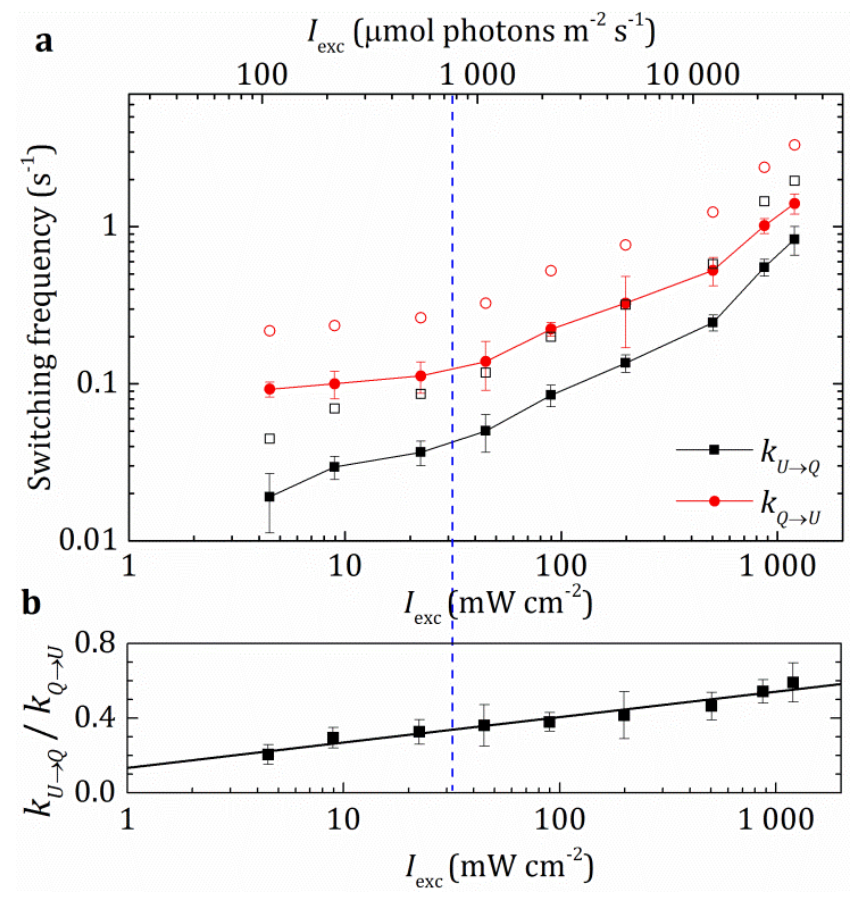

Figure 4. Intensity dependence of switching frequency between unquenched and quenched states.

a, Average switching frequency into a quenched state (black squares) and into an unquenched state (red circles) as a function of excitation intensity, using 200-ms time bins. $k_{U \rightarrow Q}$ and $k_{Q \rightarrow U}$ were calculated from Fig. S5a and b, respectively, by taking the ratio of the area under each curve and the total dwell time in unquenched (for a) or quenched (for b) states (see SI for more information). Open circles are switching rates for 10-ms binned data: the values corresponding to the two highest intensities are calculated and the average increase is extrapolated to lower intensities. The dashed line corresponds to the high-light photon flux in the pulseamplitude modulation (PAM) experiment in Fig. 5. Error bars are the sum of the standard deviation and estimated analysis uncertainty - see Supporting Experimental Information. Number of measured complexes after data screening is 369 , giving an average of 41 complexes per environment. b, Ratio of switching frequencies in a. The straight line fit regarded the square of the reciprocal of the error margins.

\section{DISCUSSION}

Provided that in the cells of cyanobacteria the equilibrium between the states also shifts toward quenched states under excessive illumination, its signature is expected in ensemble experiments in vivo in the whole, intact cells. However, in the wild-type strain of Synechocystis, the large amplitude of fluorescence quenching related to the organism's main photoprotective mechanisms - OCP-driven energy quenching - overwhelms the signatures of other mechanisms. ${ }^{16}$ Hence, to overcome this limitation we investigated whole cells of the Synechocystis OCP-depleted mutant ${ }^{16}$ in a few independent experiments and, surprisingly, we consistently observed a con- comitant quenching of the maximal fluorescence $\left(F_{m}{ }^{\prime}\right)$ and the steady-state fluorescence $\left(F_{s}\right)$ under relatively intense illumination followed by a fast fluorescence increase in dim light (Fig. 5, inset). In particular, the initial phase of fluorescence quenching (between $50 \mathrm{~s}$ and 100 s; Fig. 5) cannot be explained using previously proposed molecular mechanisms: the OCP-related mechanism is absent in this mutant, and photoinhibition would not be followed by recovery of the fluorescence directly after dimming the light at $260 \mathrm{~s}$. However, the observation that the fluorescence did not recover to the initial level does point to photoinhibition, typically signified by a linear decrease of fluorescence intensity, in this case after the initial phase of fast fluorescence quenching (i.e., photoinhibition most likely occurred between $100 \mathrm{~s}$ and $260 \mathrm{~s}$;). In addition, the involvement of state transitions, the mechanisms balancing excitation rates between the two photosystems in response to light treatment, could likely be excluded because the quenching was induced by strong blue light preceded by the dim blue light illumination (up to $50 \mathrm{~s}$ ) that set the cells in a given state (Fig. 5, inset). We therefore conclude that the fluorescence decrease displayed in Fig. 5 is related to quenching mechanism(s) within $\mathrm{PB}$ and not in chlorophyll-containing complexes, in agreement with the previously reported spectrally resolved light-activated fluorescence decrease in Synechocystis PB. ${ }^{31}$ Furthermore, the trend in Fig. 4 extending across a broad range of excitation intensities shows no indication of an additional energy-dissipation process being switched on at higher excitation intensities but instead points to an equilibrium shift favoring quenched states as the incident light intensity increases. It is therefore reasonable to assume that the mechanism responsible for light-activated fluorescence quenching described in the present study is the same mechanism that is activated in vivo.

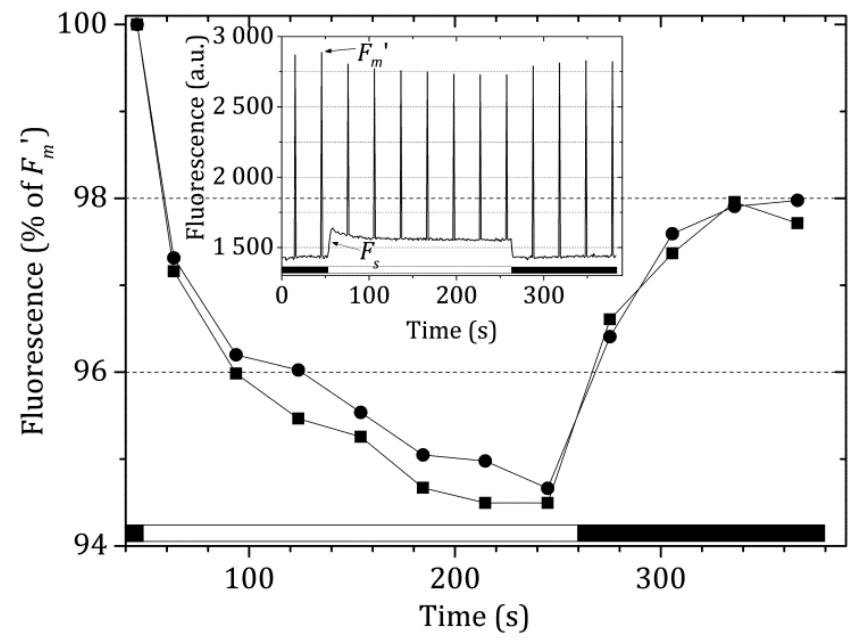

Figure 5. Two representative $F_{m}$ ' quenching and recovery traces of Synechocystis OCP-depleted mutant.

Traces extracted from two independent PAM measurements. An example corresponding to the squares is shown in the inset. Horizontal black and white bars indicate illumination at 80 and $740 \mu \mathrm{mol}$ photons $\mathrm{m}^{-2} \mathrm{~s}^{-1}$, respectively. 
The observation that nearly 3/4 of the strongly redshifted states of $\mathrm{PB}$ were preceded by blue-shifted quenched states suggests some relationship in their underlying molecular mechanisms. In the chlorophyllcontaining photosynthetic complexes, the considerably red-shifted emission arises from charge-transfer (CT) states mixing with the lowest excitonic states. ${ }^{32,33}$ By analogy, a significant charge redistribution may explain the substantially red-shifted emission from PB complexes (Fig. 2c, top spectrum, peak at $\sim 712 \mathrm{~nm}$, and Fig. S1, peak at $\sim 727 \mathrm{~nm}$ ); however, the formation of a CT state between two phycobilins in PB is unlikely because of their large spatial separation (the smallest distance being 2.1 $\mathrm{nm}) .^{34}$ Instead, we propose the energy trap to involve an intramolecular CT state within a phycobilin, possibly resulting from a configurational change, as evidenced for bilins in phytochromes. ${ }^{35,36}$ Alternatively, a local structural defect, such as a charge in a hydrophobic pocket, could alter the CT character of a pigment. Although local heat could partially trigger the recovery from a quenched state, thermally induced quenching seems improbable, as indicated in the Supporting Calculations. Other possible mechanisms can be ruled out based on insufficiently short lifetimes or insensitivity to the excitation flux. For example, non-emissive, radical states have lifetimes of only $10 \mu \mathrm{s},{ }^{37}$ and for the range of excitation intensities used in this study the yield of intersystem crossing was negligible (see Fig. $S_{3}$, where the data points follow the calculations assuming the absence of triplet states). Furthermore, rotation of a complex occurs typically on a timescale of tens of $\mu$ s and corresponds only to small intensity fluctuations. Finally, diffusion of free fractions of complexes through the excitation focal volume would result in intensity spikes due to $\mathrm{ms}$ diffusion times through the focal volume. Such intensity spikes were not observed.

If the $\mathrm{PB}$ fluorescence quenching described in this study is activated in the intact cells with a similar light dependence, this mechanism belongs to a novel class of light-activated mechanisms and is most suitable to provide immediate photoprotection when an organism is exposed to strong sunlight. It is likely that the $\sim 2 \%$ fast quenching at the particular conditions used for Fig. 5 would be sufficient for the organism until OCPdependent mechanisms are switched on. In the naturally occurring OCP-depleted strains of cyanobacteria or in red algae, this mechanism could serve as even more critical photoprotection and the extent of light-induced quenching may therefore be more pronounced. Since the energy quenching mechanism described in this study is derived from fundamental properties of phycobilins embedded in protein matrices we expect PBs of other organisms to exhibit a very similar behavior, despite differences in architecture and composition between the hemidiscoidal PBs of cyanobacteria and the phycoerythrin-rich hemiellipsoidal PBs of red algae. The presence of phycoerythrin in red algae may contribute to additional regulation of excitation energy flow, such as light-induced energetic decoupling proposed in an intensity-dependent photo- bleaching study conducted under extreme light intensities. $^{38}$

It is worth noting that the energy quenching rates of $(30-40 \mathrm{ps})^{-1}$, derived from the shortest fluorescence lifetimes measured in this study for individual $\mathrm{PB}$, compete well with the excitation energy transfer rate of $(63 \mathrm{ps})^{-1}$ from $\mathrm{PB}$ to the photosystems in vivo ${ }^{21}$ and that the excitation will reside a considerably longer time in the antenna when the reaction center is closed.

\section{CONCLUSION}

The size of PB not only enables more efficient absorption under low light conditions but it also lends the organism a more efficient response to rapidly changing light conditions by providing room for numerous quenching centers. From this study we conclude that the probability of accessing one or more of these quenching centers is governed by the conformational dynamics, which in turn is subtly controlled by the mere photon flux. As such, we demonstrated that cyanobacterial antennae have an intrinsic, fast, light-controlled, and highly sensitive and effective regulatory mechanism, and it is very likely that other photosynthetic organisms use a similar strategy for self-protection against large and rapid intensity fluctuations. The ability of a system to modulate non-radiative energy dissipation using only light is a property of great potential for solar energy and food technologies. For example, precise regulation of the excitation energy flow in bioinspired devices is required for the effective conversion of solar energy.

\section{EXPERIMENTAL SECTION SUMMARY}

Isolation of PB. Intact PB as well as PB cores and cores with short rods, and rod fractions were purified from WT Synechocystis and $\mathrm{CK},{ }^{29} \mathrm{CB},{ }^{28}$ and $\Delta \mathrm{AB}^{27}$ mutants as reported before in Ref. 19, The samples were prepared for the single molecule experiments by a means of serial dilution and immobilization on a microscope cover glass (see SI for details). For the experiment with the cross-linker, Glutaraldehyde (Sigma) was added once the complexes were immobilized on a microscope cover glass.

Single molecule spectroscopy and data analysis. The measurements were performed as previously described in Ref. 33, with some sample- and experimentspecific modifications. See SI for the details as well as for information on data analysis and modeling.

Pulse-amplitude modulation (PAM) measurement. PAM measurements on Synechocystis OCP-depleted mu$\operatorname{tant}^{16}$ were repeated 6 times and performed as described in Ref. 16 (with two repetitions shown in Fig. 5). Low blue light intensity used in the measurement before and after the quenching was at $80 \mu \mathrm{mol}$ photons $\mathrm{m}^{-2} \mathrm{~s}^{-1}$, while the high blue light intensity inducing quenching was at 740 $\mu \mathrm{mol}$ photons $\mathrm{m}^{-2} \mathrm{~s}^{-1}$. 


\section{ASSOCIATED CONTENT}

Supporting Information for Publication composed of Supporting Figures, Table, Calculations, Experimental Information, and References. This material is available free of charge via the Internet at http://pubs.acs.org.

\section{AUTHOR INFORMATION}

\section{Corresponding Authors}

* Michal Gwizdala

m.s.gwizdala@vu.nl

* Tjaart P.J. Krüger

tjaart.kruger@up.ac.za

\section{Present Addresses}

†: Michal Gwizdala

Department of Physics,

University of Pretoria,

Private bag X20, Hatfield oo28, South Africa

E-mail: gwizdala.michal@gmail.com

Tel: +276o 7175821

\section{Author Contributions}

All authors have given approval to the final version of the manuscript.

The authors declare no competing financial interest.

\section{ACKNOWLEDGMENT}

We thank Adjélé Wilson, Sandrine Cot and J. Michael Gruber for technical help. MG acknowledges the European Molecular Biology Organization for funding his Long Term Fellowship. The work of MG, TPJK, RB and RvG was supported from advanced investigator grant (267333, PHOTPROT) to RvG from the European Research Council and the TOP grant (700.58.305) from the Foundation of Chemical Sciences part of NWO. RvG gratefully acknowledges his 'Academy Professor' grant from the Royal Netherlands Academy of Arts and Sciences (KNAW). TPJK was further supported by the University of Pretoria's Research Development Programme (Grant No. AoW679). RB also kindly acknowledges financial support from the Japanese Society for the Promotion of Science (JSPS) via a long-term visitor grant. The work in the laboratory of DK was supported from Agence Nationale de la Recherche (project CYANOPROTECT), the CNRS, the Commissariat à l'Energie Atomique, the HARVEST EU FP 7 Marie Curie Research Training Network, Phycosource, and the French Infrastructure for Integrated Structural Biology (Grant ANR-10-INSB-05-01).

\section{REFERENCES}

(1) Ruban, A. V. J. Exp. Bot. 2o14, 66, 7-23
(2) Demmig-Adams, B.; Garab, G.; Adams III, W.W.; Govindjee. 2014, 40, Springer, Dordrecht, The Netherlands.

(3) van Grondelle, R.; Dekker, J. P.; Gillbro, T.; Sundstrom, V. BBA-Bioenergetics 1994, 1187, 1-65.

(4) Foyer, C. H.; Lelandais, M.; Kunert, K. J. Physiol. Plantarum 1994, 92, 696-717.

(5) Cichos, F.; von Borczyskowski, C.; Orrit, M. Curr. Opin. Colloid In. 2007, 12, 272-284.

(6) Krüger, T. P. J.; Ilioaia, C.; Johnson, M. P.; Ruban, A. V.; Papagiannakis, E.; Horton, P.; van Grondelle, R. Biophys. J. 2012, 102, 2669-2676.

(7) Valkunas, L.; Chmeliov, J.; Krüger, T. P. J.; Ilioaia, C.; van Grondelle, R. J. Phys. Chem. Lett. 2o12, 3, 2779-2784.

(8) Krüger, T. P. J.; Ilioaia, C.; Valkunas, L.; van Grondelle, R. J. Phys. Chem. B 2011, 115, 5083-5095.

(9) Gruber, J. M.; Chmeliov, J.; Krüger, T. P. J.; Valkunas, L.; van Grondelle. R. Phys. Chem. Chem. Phys. 2015, 17, 19844-19853.

(10) Zondervan, R.; Kulzer, F.; Orlinskii, S. B.; Orrit, M. J. Phys. Chem. A 2003, 107, 6770-6776.

(11) Glazer, A. N. Biochim. Biophys. Acta 1984, 768, 2951.

(12) Arteni, A.; Ajlani, G.; Boekema, E. $B B A-$ Bioenergetics 2009, 1787, 272-279.

(13) Liu, H.; Zhang, H.; Niedzwiedzki, D. M.; Prado, M.; He, G.; Gross, M. L.; Blankenship, R. E. Science 2013, 342, 1104-1107.

(14) Adir, N. Photosynth. Res. 2005, 85, 15-32.

(15) Lundell, D. J.; Glazer, A. N. J. Biol. Chem. 1983, 258, $8708-8713$.

(16) Wilson, A.; Ajlani, G.; Verbavatz, J.-M.; Vass, I.; Kerfeld, C. A.; Kirilovsky, D. Plant Cell 20o6, 18, 992-1007.

(17) Wilson, A.; Punginelli, C.; Gall, A.; Bonetti, C.; Alexandre, M.; Routaboul, J.-M.; Kerfeld, C. A.; van Grondelle, R.; Robert, B.; Kennis, J. T. M.; Kirilovsky, D. P. Natl. Acad. Sci. USA 2008, 105, 1207512080 .

(18) Leverenz, R. L.; Sutter, M.; Wilson, A.; Gupta, S.; Thurotte, A.; Bourcier de Carbon, C.; Petzold, C. J.; Ralston, C.; Perreau, F.; Kirilovsky, D.; Kerfeld, C. A. Science 2015, 348, 1463-1466. 
(19) Gwizdala, M.; Wilson, A.; Kirilovsky, D. Plant Cell 2011, 23, 2631-2643.

(20)Harris, D.; Tal, O.; Jallet, D.; Wilson, A.; Kirilovsky, D.; Adir, N. P. Natl. Acad. Sci. USA 2016, 113, 16551662.

(21) Tian, L.; van Stokkum, I. H. M.; Koehorst, R. B. M.; Jongerius, A.; Kirilovsky, D.; van Amerongen, H. J. Am. Chem. Soc. 2011, 133, 18304-18311.

(22) Jallet, D.; Gwizdala, M.; Kirilovsky, D. BBABioenergetics 2012, 1817, 1418-1427.

(23) Gantt, E.; Lipschultz, C. A. J. Cell Biol. 1972, 54, 313324.

(24)Schlau-Cohen, G. S.; Yang, H. Y.; Krüger, T. P. J.; $\mathrm{Xu}$, P.; Gwizdala, M.; van Grondelle, R.; Croce, R.; Moerner W. E J. Phys. Chem. Lett. 2015, 6, 86o867.

(25) Loos, D.; Cotlet, M.; De Schryver, F.; Habuchi, S.; Hofkens, J. Biophys. J. 2004, 87, 2598-26o8.

(26)Goldsmith, R. H.; Moerner W. E. Nature Chem. 2010, 2, 179-186.

(27) Ajlani, G.; Vernotte, C.; DiMagno, L.; Haselkorn, R. BBA-Bioenergetics 1995, 1231, 189-196.

(28)Ughy, B.; Ajlani, G. Microbiology 2004, 150, 41474156.

(29)Piven, I.; Ajlani, G.; Sokolenko, A. J. Biol. Chem. 2005, 280, 21667-21672.
(30)Wang, Q.; Moerner, W. E. P. Natl. Acad. Sci. USA 2015, 112, 13880-13885.

(31) Stoitchkova, K.; Zsiros, O.; Jávorfi, T.; Páli, T.; Andreeva, A.; Gombos, Z.; Garab, G. BBABioenergetics 2007, 1767, 750-756.

(32) Romero, E.; Mozzo, M.; van Stokkum, I. H. M.; Dekker, J. P.; van Grondelle, R.; Croce, R. Biophys. J. 2009, 96, L35-37.

(33) Krüger, T. P. J.; Novoderezhkin, V. I.; Ilioaia, C.; van Grondelle, R. Biophys. J. 2010, 98, 3093-3101.

(34)Reuter, W.; Wiegand, G.; Huber, R.; Than, M. P. Natl. Acad. Sci. USA 1999, 96, 1363-1368.

(35) Fodor, S. P. A.; Lagarias, J. C.; Mathies, R. A. Biochemistry-US 1990, 29, 11141-11146.

(36)Ulijasz, A. T.; Cornilescu, G.; Cornilescu, C. C.; Zhang, J.; Rivera, M.; Markley, J. L.; Vierstra, R. D. Nature 2010, 463, 250-254.

(37)Zhang, S. P.; Zhao, J. Q.; Jiang, L. J. Free Radial Res. 2000, 33, 489-496.

(38)Liu, L.-N.; Elmalk, A. T.; Aartsma, T. J.; Thomas, J.C.; Lamers, G. E. M.; Zhou, B.-C.; Zhang, Y.-Z. PLoS One 2008, 3, e3134 